\title{
Lax Integrable Supersymmetric Hierarchies on Extended Phase Spaces
}

\author{
Oksana Ye. HENTOSH \\ Institute for Applied Problems of Mechanics and Mathematics, \\ National Academy of Sciences of Ukraine, 3B Naukova Str., Lviv, 79060 Ukraine \\ E-mail: dept25@iapmm.lviv.ua
}

Received October 27, 2005, in final form December 21, 2005; Published online January 04, 2006

Original article is available at http://www.emis.de/journals/SIGMA/2006/Paper001/

\begin{abstract}
We obtain via Bäcklund transformation the Hamiltonian representation for a Lax type nonlinear dynamical system hierarchy on a dual space to the Lie algebra of super-integral-differential operators of one anticommuting variable, extended by evolutions of the corresponding spectral problem eigenfunctions and adjoint eigenfunctions, as well as for the hierarchies of their additional symmetries. The relation of these hierarchies with the integrable by Lax $(2 \mid 1+1)$-dimensional supersymmetric Davey-Stewartson system is investigated.
\end{abstract}

Key words: Lax type flows; "ghost" symmetries; the Davey-Stewartson system

2000 Mathematics Subject Classification: 35Q53; 35Q58; 37K10; 37K30; 37K35; 58A50

\section{Introduction}

Since the paper of M. Adler [1] there was an understanding that Lax forms for a wide class of integrable nonlinear dynamical system hierarchies on functional manifolds $[2,3,4,5]$ and their supersymmetric analogs $[6,7]$ could be considered as Hamiltonian flows on dual spaces to the Lie algebra of integro-differential operators. Those flows are generated by the $\mathcal{R}$-deformed canonical Lie-Poisson bracket and Casimir functionals as Hamiltonian functions (see $[1,8,9]$ ). For a concrete integro-differential operator every Hamiltonian flow of such a type can be written as a compatibility condition for the corresponding isospectral problem in the case of an arbitrary eigenfunction and the suitable evolution of this function. Thus, the existence problem of a Hamiltonian representation for the Lax type hierarchy, extended by the evolutions of a finite set of eigenfunctions and appropriate adjoint eigenfunctions, arises. In $[10,11,12]$ it was solved for the Lie algebra of integro-differential operators by use of the Casimir functionals' invariant property under some Lie-Bäcklund transformation. Analogously we obtain in this paper the Hamiltonian representation of the extended Lax type system hierarchy for the Lie algebra of super-integro-differential operators of one anticommuting variable.

The hierarchies of additional or "ghost" symmetries [13] for the extended Lax type system are also proved to be Hamiltonian. It is established that every additional symmetry hierarchy is generated by the tensor product of the $\mathcal{R}$-deformed canonical Lie-Poisson bracket with the Poisson bracket on a finite-dimensional superspace, possessing an odd supersymplectic structure [14, 15], and all natural powers of one eigenvalue from the mentioned above finite set as Hamiltonian functions. The additional symmetry hierarchy is used for introducing one more commuting variable into $(1 \mid 1+1)$-dimensional supersymmetric nonlinear dynamical systems with preserving their integrability by Lax. By means of this approach a $(2 \mid 1+1)$-dimensional supersymmetric analog of the Davey-Stewartson system $[5,16,17]$ and its triple linearization of a Lax type are found. 


\section{The general algebraic scheme}

Let $\mathcal{G}$ be a Lie algebra of scalar super-integral-differential operators [6] of one anticommuting variable $\theta\left(\theta^{2}=0\right)$ :

$$
a:=\partial^{m}+\sum_{j<2 m} a_{j} D_{\theta}^{j}, \quad m \in \mathbb{N},
$$

where the symbol $\partial:=\partial / \partial x$ designates differentiation with respect to the independent variable $x \in \mathbb{R} / 2 \pi \mathbb{Z} \simeq \mathbb{S}^{1}, a_{j}:=a_{j}(x, \theta)=a_{j}^{0}(x)+\theta a_{j}^{1}(x), j \in \mathbb{Z}$, are smooth superfield functions (superfunctions), and the superderivative $D_{\theta}:=\partial / \partial \theta+\theta \partial / \partial x$, for which $D_{\theta}^{2}=\partial$, satisfies the following relation for any smooth superfield functions $u$ and $v$ :

$$
D_{\theta}(u v)=\left(D_{\theta} u\right) v+(-1)^{p(u)} u\left(D_{\theta} v\right)
$$

where $p(u)$ is a parity of an arbitrary superfunction $u$, which is equal to 0 for $u$, being even, and one for $u$, being odd.

The usual Lie commutator on $\mathcal{G}$ is defined as

$$
[a, b]:=a \circ b-b \circ a
$$

for all $a, b \in \mathcal{G}$, where "o" is an associative product of super-integro-differential operators. On the Lie algebra $\mathcal{G}$ there exists the $a d$-invariant nondegerated symmetric bilinear form:

$$
(a, b):=\int_{0}^{2 \pi} \operatorname{res}_{D_{\theta}}(a \circ b) d x
$$

where $\operatorname{res}_{D_{\theta}}$-operation for all $a \in \mathcal{G}$ is given by the expression:

$$
\operatorname{res}_{D_{\theta}} a:=a_{-1} .
$$

By means of the scalar product (1) the Lie algebra $\mathcal{G}$ is transformed into a metrizable one. As a consequence, its dual linear space of scalar super-integro-differential operators $\mathcal{G}^{*}$ is identified with the Lie algebra $\mathcal{G}$, that is $\mathcal{G}^{*} \simeq \mathcal{G}$.

The linear subspaces $\mathcal{G}_{+} \subset \mathcal{G}$ and $\mathcal{G}_{-} \subset \mathcal{G}$

$$
\begin{aligned}
& \mathcal{G}_{+}:=\left\{a:=\partial^{m}+\sum_{j=0}^{2 m-1} a_{j} D_{\theta}^{j}: j=\overline{0,2 m-1}\right\}, \\
& \mathcal{G}_{-}:=\left\{b:=\sum_{l>0}^{\infty} b_{l} D_{\theta}^{-l}: l \in \mathbb{N}\right\},
\end{aligned}
$$

where $a_{j}$ and $b_{l}$ are smooth superfunctions, forms Lie subalgebras in $\mathcal{G}$ and $\mathcal{G}=\mathcal{G}_{+} \oplus \mathcal{G}_{-}$. Because of the splitting of $\mathcal{G}$ into the direct sum (2) of its Lie subalgebras one can construct a Lie-Poisson structure $[1,8,9]$ on $\mathcal{G}^{*}$, using the special linear endomorphism $\mathcal{R}$ of $\mathcal{G}$ :

$$
\mathcal{R}:=\left(P_{+}-P_{-}\right) / 2, \quad P_{ \pm} \mathcal{G}:=\mathcal{G}_{ \pm}, \quad P_{ \pm} \mathcal{G}_{\mp}=0 .
$$

For any smooth by Frechet functionals $\gamma, \mu \in \mathcal{D}\left(\mathcal{G}^{*}\right)$ the Lie-Poisson bracket on $\mathcal{G}^{*}$ is given by the expression:

$$
\{\gamma, \mu\}_{\mathcal{R}}(l)=\left(l,[\nabla \gamma(l), \nabla \mu(l)]_{\mathcal{R}}\right),
$$


where $l \in \mathcal{G}^{*}$ and for all $a, b \in \mathcal{G}$ the $\mathcal{R}$-deformed commutator has the form:

$$
[a, b]_{\mathcal{R}}:=[\mathcal{R} a, b]+[a, \mathcal{R} b] .
$$

The linear space $\mathcal{G}$ with the commutator (4) also becomes a Lie algebra. The gradient $\nabla \gamma(l) \in \mathcal{G}$ of some functional $\gamma \in \mathcal{D}\left(\mathcal{G}^{*}\right)$ at the point $l \in \mathcal{G}^{*}$ with respect to the scalar product (1) is defined as

$$
\delta \gamma(l):=(\nabla \gamma(l), \delta l),
$$

where the linear space isomorphism $\mathcal{G} \simeq \mathcal{G}^{*}$ is taken into account.

Every Casimir functional $\gamma \in I\left(G^{*}\right)$, being invariant with respect to $\mathrm{Ad}^{*}$-action of the corresponding Lie group $G$, obeys the following condition at the point $l \in \mathcal{G}^{*}$ :

$$
[l, \nabla \gamma(l)]=0 .
$$

The relationship (5) is satisfied by the hierarchy of functionals $\gamma_{n} \in I\left(G^{*}\right), n \in \mathbb{Z}_{+}$, taking the forms:

$$
\gamma_{n}(l)=\frac{1}{n+1}\left(l^{1 / m}, l^{n / m}\right) .
$$

The Lie-Poisson bracket (3) generates the hierarchy of Hamiltonian dynamical systems on $\mathcal{G}^{*}$ :

$$
d l / d t_{n}:=\left[\mathcal{R} \nabla \gamma_{n}(l), l\right]=\left[\left(\nabla \gamma_{n}(l)\right)_{+}, l\right],
$$

with the Casimir functionals (6) as Hamiltonian functions.

The latter equation is equivalent to the usual commutator Lax type representation. It is easy to verify that for every $n \in \mathbb{Z}_{+}$the relationship (7) is a compatibility condition for such linear integral-differential equations:

$$
l f=\lambda f,
$$

and

$$
d f / d t_{n}=\left(\nabla \gamma_{n}(l)\right)_{+} f
$$

where $\lambda \in \mathbb{C}$ is a spectral parameter, $f \in W^{1 \mid 0}:=L_{\infty}\left(\mathbb{S}^{1} \times \Lambda_{1} ; \mathbb{C}^{1 \mid 0}\right)$ if $f$ is an even superfunction and $f \in W^{0 \mid 1}:=L_{\infty}\left(\mathbb{S}^{1} \times \Lambda_{1} ; \mathbb{C}^{0 \mid 1}\right)$ if $f$ is an odd one. Here $\Lambda:=\Lambda_{0} \oplus \Lambda_{1}$ is a Grassmann algebra over $\mathbb{C}, \Lambda_{0} \supset \mathbb{R}$. The associated with (9) dynamical system for the adjoint superfunction $f^{*}$ takes the form:

$$
d f^{*} / d t_{n}=-\left(\nabla \gamma_{n}(l)\right)_{+}^{*} f^{*}
$$

where $\left(f, f^{*}\right)^{T} \in W^{1 \mid 1}:=L_{\infty}\left(\mathbb{S}^{1} \times \Lambda_{1} ; \mathbb{C}^{1 \mid 1}\right)$ or $\left(f^{*}, f\right)^{T} \in W^{1 \mid 1}$ and superfunction $f^{*}$ is a solution of the adjoint spectral problem:

$$
l^{*} f^{*}=\lambda f^{*} .
$$

The objects of further investigations are some algebraic properties of equation (7) together with $2 N \in \mathbb{N}$ copies of equation (9):

$$
\begin{aligned}
& d f_{i} / d t_{n}=\left(\nabla \gamma_{n}(l)\right)_{+} f_{i}, \\
& d \Phi_{i} / d t_{n}=\left(\nabla \gamma_{n}(l)\right)_{+} \Phi_{i},
\end{aligned}
$$

for even $f_{i} \in W^{1 \mid 0}$ and odd $\Phi_{i} \in W^{0 \mid 1}$ eigenfunctions of the spectral problem (8), corresponding to the eigenvalues $\lambda_{i}, i=\overline{1, N}$, and the same number of copies of equation (10):

$$
\begin{aligned}
& d f_{i}^{*} / d t_{n}=-\left(\nabla \gamma_{n}(l)\right)_{+}^{*} f_{i}^{*}, \\
& d \Phi_{i}^{*} / d t_{n}=-\left(\nabla \gamma_{n}(l)\right)_{+}^{*} \Phi_{i}^{*},
\end{aligned}
$$

for corresponding odd $f_{i}^{*} \in W^{0 \mid 1}$ and even $\Phi_{i}^{*} \in W^{1 \mid 0}$ adjoint eigenfunctions, as a coupled evolution system on the space $\mathcal{G}^{*} \oplus W^{2 N \mid 2 N}$. 


\section{Tensor product of Poisson structures and its Bäcklund transformation}

To compactify the description below one shall use the following designation of the left gradient vector:

$$
\nabla \gamma\left(\tilde{l}, \tilde{f}_{i}, \tilde{\Phi}_{i}^{*}, \tilde{f}_{i}^{*}, \tilde{\Phi}_{i}\right):=\left(\frac{\delta \gamma}{\delta \tilde{l}}, \frac{\delta \gamma}{\delta \tilde{f}_{i}}, \frac{\delta \gamma}{\delta \tilde{\Phi}_{i}^{*}}, \frac{\delta \gamma}{\delta \tilde{f}_{i}^{*}}, \frac{\delta \gamma}{\delta \tilde{\Phi}_{i}}\right)^{T}
$$

where $i=\overline{1, N}$, at a point $\left(\tilde{l}, \tilde{f}_{i}, \tilde{\Phi}_{i}^{*}, \tilde{f}_{i}^{*}, \tilde{\Phi}_{i}\right)^{T} \in \mathcal{G}^{*} \oplus W^{2 N \mid 2 N}$ for any smooth functional $\gamma \in$ $\mathcal{D}\left(\mathcal{G}^{*} \oplus W^{2 N \mid 2 N}\right)$.

On the spaces $\mathcal{G}^{*}$ and $W^{N} \oplus W^{* N}$ there exist a Lie-Poisson structure [1, 8, 9]

$$
\delta \gamma / \delta \tilde{l}: \stackrel{\tilde{\Theta}}{\rightarrow}\left[\tilde{l},\left(\frac{\delta \gamma}{\delta \tilde{l}}\right)_{+}\right]-\left[\tilde{l}, \frac{\delta \gamma}{\delta \tilde{l}}\right]_{+},
$$

where $\tilde{\Theta}: \mathcal{G} \rightarrow \mathcal{G}^{*}$, at a point $\tilde{l} \in \mathcal{G}^{*}$ and the canonical Poisson structure $[14,15]$

$$
\left(\frac{\delta \gamma}{\delta \tilde{f}_{i}}, \frac{\delta \gamma}{\delta \tilde{\Phi}_{i}^{*}}, \frac{\delta \gamma}{\delta \tilde{f}_{i}^{*}}, \frac{\delta \gamma}{\delta \tilde{\Phi}_{i}}\right)^{T}: \stackrel{\tilde{J}}{\rightarrow}\left(-\frac{\delta \gamma}{\delta \tilde{f}_{i}^{*}}, \frac{\delta \gamma}{\delta \tilde{\Phi}_{i}}, \frac{\delta \gamma}{\delta \tilde{f}_{i}},-\frac{\delta \gamma}{\delta \tilde{\Phi}_{i}^{*}},\right)^{T}
$$

$\tilde{J}: T^{*}\left(W^{2 N \mid 2 N}\right) \rightarrow T\left(W^{2 N \mid 2 N}\right)$, corresponding to the odd symplectic form $\omega^{(2)}=\sum_{i=1}^{N}\left(\tilde{f}_{i} \wedge \tilde{f}_{i}^{*}-\right.$ $\left.\tilde{\Phi}_{i} \wedge \tilde{\Phi}_{i}^{*}\right)$, at a point $\left(\tilde{f}_{i}, \tilde{\Phi}_{i}^{*}, \tilde{f}_{i}^{*}, \tilde{\Phi}\right)^{T} \in W^{2 N \mid 2 N}$. It should be noted that the Poisson structure (13) generates equation (7) for any Casimir functional $\gamma \in I\left(\mathcal{G}^{*}\right)$.

Thus, on the extended phase space $\mathcal{G}^{*} \oplus W^{2 N \mid 2 N}$ one can obtain a Poisson structure as the tensor product $\tilde{\mathcal{L}}:=\tilde{\Theta} \otimes \tilde{J}$ of $(13)$ and $(14)$.

Consider the following Bäcklund transformation:

$$
\left(\tilde{l}, \tilde{f}_{i}, \tilde{\Phi}_{i}^{*}, \tilde{f}_{i}^{*}, \tilde{\Phi}_{i}\right)^{T}: \stackrel{B}{\mapsto}\left(l\left(\tilde{l}, \tilde{f}_{i}, \tilde{\Phi}_{i}^{*}, \tilde{f}_{i}^{*}, \tilde{\Phi}_{i}\right), f_{i}=\tilde{f}_{i}, \Phi_{i}^{*}=\tilde{\Phi}_{i}^{*}, f_{i}^{*}=\tilde{f}_{i}^{*}, \Phi_{i}=\tilde{\Phi}_{i}\right)^{T}
$$

generating on $\mathcal{G}^{*} \oplus W^{2 N \mid 2 N}$ a Poisson structure $\mathcal{L}$ with respect to variables $\left(l, f_{i}, \Phi_{i}^{*}, f_{i}^{*}, \Phi_{i}\right)$, $i=\overline{1, N}$, of the coupled evolution equations (7), (11) and (12). The main condition for the mapping (15) is coincidence of the dynamical system

$$
\left(d l / d t, d f_{i} / d t, d \Phi_{i}^{*} / d t, d f_{i}^{*} / d t, d \Phi_{i} / d t\right)^{T}:=-\mathcal{L} \nabla \gamma_{n}\left(l, f_{i}, \Phi_{i}^{*}, f_{i}^{*}, \Phi_{i}\right)
$$

with equations (7), (11) and (12) in the case of $\gamma_{n} \in I\left(\mathcal{G}^{*}\right), n \in \mathbb{Z}_{+}$, i.e. when the functional $\gamma_{n}$ is taken to be not dependent of variables $\left(f_{i}, \Phi_{i}^{*}, f_{i}^{*}, \Phi_{i}\right)^{T} \in W^{2 N \mid 2 N}$. To satisfy that condition, one should find a variation of some Casimir functional $\gamma_{n} \in I\left(\mathcal{G}^{*}\right), n \in \mathbb{Z}_{+}$, at $\delta \tilde{l}=0$, taking into account the evolutions (11), (12) and the Bäcklund transformation (15):

$$
\begin{aligned}
\left.\delta \gamma_{n}\left(\tilde{l}, \tilde{f}_{i}, \tilde{\Phi}_{i}^{*}, \tilde{f}_{i}^{*}, \tilde{\Phi}_{i}\right)\right|_{\delta \tilde{l}=0} \\
=\sum_{i=1}^{N}\left(\left\langle\delta \tilde{f}_{i}, \frac{\delta \gamma_{n}}{\delta \tilde{f}_{i}}\right\rangle+\left\langle\delta \tilde{\Phi}_{i}^{*}, \frac{\delta \gamma_{n}}{\delta \tilde{\Phi}_{i}^{*}}\right\rangle+\left\langle\delta \tilde{f}_{i}^{*}, \frac{\delta \gamma_{n}}{\delta \tilde{f}_{i}^{*}}\right\rangle+\left\langle\delta \tilde{\Phi}_{i}, \frac{\delta \gamma_{n}}{\delta \tilde{\Phi}_{i}}\right\rangle\right) \\
=\sum_{i=1}^{N}\left(\left\langle\delta \tilde{f}_{i},-\frac{d \tilde{f}_{i}^{*}}{d t_{n}}\right\rangle+\left\langle\delta \tilde{\Phi}_{i}^{*}, \frac{d \tilde{\Phi}_{i}}{d t_{n}}\right\rangle+\left\langle\delta \tilde{f}_{i}^{*}, d \tilde{f}_{i} / d t_{n}\right\rangle+\left\langle\delta \tilde{\Phi}_{i},-\frac{d \tilde{\Phi}_{i}^{*}}{d t_{n}}\right\rangle\right) \\
=\sum_{i=1}^{N}\left(\left\langle\delta f_{i},\left(\nabla \gamma_{n}(l)\right)_{+}^{*} f_{i}^{*}\right\rangle+\left\langle\delta \Phi_{i}^{*},\left(\nabla \gamma_{n}(l)\right)_{+} \Phi_{i}\right\rangle+\left\langle\delta f_{i}^{*},\left(\nabla \gamma_{n}(l)\right)_{+} f_{i}\right\rangle\right.
\end{aligned}
$$




$$
\begin{aligned}
& \left.+\left\langle\delta \Phi_{i},\left(\nabla \gamma_{n}(l)\right)_{+}^{*} \Phi_{i}^{*}\right\rangle\right)=\sum_{i=1}^{N}\left(\left\langle\left(\nabla \gamma_{n}(l)\right)_{+} \delta f_{i}, f_{i}^{*}\right\rangle+\left\langle\left(\nabla \gamma_{n}(l)\right)_{+} f_{i}, \delta f_{i}^{*}\right\rangle\right. \\
& \left.+\left\langle\left(\nabla \gamma_{n}(l)\right)_{+}\left(\delta \Phi_{i}\right), \Phi_{i}^{*}\right\rangle+\left\langle\left(\nabla \gamma_{n}(l)\right)_{+} \Phi_{i}, \delta \Phi_{i}^{*}\right\rangle\right) \\
& =\sum_{i=1}^{N}\left(\left(\nabla \gamma_{n}(l), \delta\left(f_{i} D_{\theta}^{-1} f_{i}^{*}\right)\right)+\left(\nabla \gamma_{n}(l), \delta\left(\Phi_{i} D_{\theta}^{-1} \Phi_{i}^{*}\right)\right)\right) \\
& =\left(\nabla \gamma_{n}(l), \delta \sum_{i=1}^{N}\left(f_{i} D_{\theta}^{-1} f_{i}^{*}+\Phi_{i} D_{\theta}^{-1} \Phi_{i}^{*}\right)\right):=\left(\nabla \gamma_{n}(l), \delta l\right),
\end{aligned}
$$

where $\gamma_{n} \in I\left(\mathcal{G}^{*}\right), n \in \mathbb{Z}_{+}$, at the point $l \in \mathcal{G}^{*}$ and the brackets $\langle\cdot, \cdot\rangle$ designate paring of the spaces $W^{1 \mid 0}$ and $W^{0 \mid 1}$. As a result of the expression (17) one obtains the relationships:

$$
\left.\delta l\right|_{\delta \tilde{l}=0}=\delta \sum_{i=1}^{N}\left(f_{i} D_{\theta}^{-1} f_{i}^{*}+\Phi_{i} D_{\theta}^{-1} \Phi_{i}^{*}\right)
$$

Having assumed the linear dependence of $l$ from $\tilde{l} \in \mathcal{G}^{*}$ one gets right away that

$$
l=\tilde{l}+\sum_{i=1}^{N}\left(f_{i} D_{\theta}^{-1} f_{i}^{*}+\Phi_{i} D_{\theta}^{-1} \Phi_{i}^{*}\right)
$$

Thus, the Bäcklund transformation (15) can be written as

$$
\left(\tilde{l}, \tilde{f}_{i}, \tilde{\Phi}_{i}^{*}, \tilde{f}_{i}^{*}, \tilde{\Phi}_{i}\right)^{T} \stackrel{B}{\mapsto}\left(l=\tilde{l}+\sum_{i=1}^{N}\left(f_{i} D_{\theta}^{-1} f_{i}^{*}+\Phi_{i} D_{\theta}^{-1} \Phi_{i}^{*}\right), f_{i}, \Phi_{i}^{*}, f_{i}^{*}, \Phi_{i}\right)^{T}
$$

The expression (19) generalizes the result obtained in the papers [10, 11, 12] for the Lie algebra of integral-differential operators. The existence of the Bäcklund transformation (19) makes it possible to formulate the following theorem.

Theorem 1. The dynamical system on $\mathcal{G}^{*} \oplus W^{2 N \mid 2 N}$, being Hamiltonian with respect to the Poisson structure $\tilde{\mathcal{L}}: T^{*}\left(\mathcal{G}^{*} \oplus W^{2 N \mid 2 N}\right) \rightarrow T\left(\mathcal{G}^{*} \oplus W^{2 N \mid 2 N}\right)$, in the form of the following evolution equations:

$$
\begin{aligned}
\frac{d \tilde{l}}{d t_{n}} & =\left[\left(\frac{\delta \gamma_{n}}{\delta \tilde{l}}\right)_{+}, \tilde{l}\right]-\left[\frac{\delta \gamma_{n}}{\delta \tilde{l}}, \tilde{l}\right]_{+}, \\
\frac{d \tilde{f}_{i}}{d t_{n}} & =\frac{\delta \gamma_{n}}{\delta \tilde{f}_{i}^{*}}, \quad \frac{d \tilde{\Phi}_{i}^{*}}{d t_{n}}=-\frac{\delta \gamma_{n}}{\delta \tilde{\Phi}_{i}}, \quad \frac{d \tilde{f}_{i}^{*}}{d t_{n}}=-\frac{\delta \gamma_{n}}{\delta \tilde{f}_{i}}, \quad \frac{d \tilde{\Phi}_{i}}{d t_{n}}=\frac{\delta \gamma_{n}}{\delta \tilde{\Phi}_{i}^{*}},
\end{aligned}
$$

where $i=\overline{1, N}$ and $\gamma_{n} \in I\left(\mathcal{G}^{*}\right), n \in \mathbb{Z}_{+}$, is a Casimir functional at the point $l \in \mathcal{G}^{*}$, connected with $\tilde{l} \in \mathcal{G}^{*}$ by (18), is equivalent to the system (9), (13) and (14) via the Bäcklund transformation (19).

By means of simple calculations via the formula:

$$
\mathcal{L}=B^{\prime} \tilde{\mathcal{L}} B^{*}
$$


where $B^{\prime}: T\left(\mathcal{G}^{*} \oplus W^{2 N \mid 2 N}\right) \rightarrow T\left(\mathcal{G}^{*} \oplus W^{2 N \mid 2 N}\right)$ is a Frechet derivative of (19), one brings about the following form of the Poisson structure $\mathcal{L}$ on $\mathcal{G}^{*} \oplus W^{2 N \mid 2 N} \ni\left(l, f_{i}, \Phi_{i}^{*}, f_{i}^{*}, \Phi_{i}\right)^{T}$ :

$$
\nabla \gamma\left(l, f_{i}, \Phi_{i}^{*}, f_{i}^{*}, \Phi_{i}\right) \stackrel{\mathcal{L}}{\rightarrow}\left(\begin{array}{c}
{\left[\tilde{l},\left(\frac{\delta \gamma}{\delta \tilde{l}}\right)_{+}\right]-\left[\tilde{l}, \frac{\delta \gamma}{\delta \tilde{l}}\right]_{+}+\sum_{i=1}^{N}\left(f_{i} D_{\theta}^{-1} \frac{\delta \gamma}{\delta f_{i}}-\right.} \\
\left.-\frac{\delta \gamma}{\delta f_{i}^{*}} D_{\theta}^{-1} f_{i}^{*}+\Phi_{i} D_{\theta}^{-1} \frac{\delta \gamma}{\delta \Phi_{i}}-\frac{\delta \gamma}{\delta \Phi_{i}^{*}} D_{\theta}^{-1} \Phi_{i}^{*}\right) \\
-\frac{\delta \gamma}{\delta f_{i}^{*}}-\left(\frac{\delta \gamma}{\delta l}\right)_{+} f_{i} \\
\frac{\delta \gamma}{\delta \Phi_{i}}+\left(\frac{\delta \gamma}{\delta l}\right)_{+}^{*} \Phi_{i}^{*} \\
\frac{\delta \gamma}{\delta f_{i}^{*}}+\left(\frac{\delta \gamma}{\delta l}\right)_{+}^{*} f_{i}^{*} \\
-\frac{\delta \gamma}{\delta \Phi_{i}^{*}}-\left(\frac{\delta \gamma}{\delta l}\right)_{+}^{+} \Phi_{i}
\end{array}\right),
$$

where $\gamma \in D\left(\mathcal{G}^{*} \oplus W^{2 N \mid 2 N}\right)$ is an arbitrary smooth functional and $i=\overline{1, N}$, that makes it possible to formulate the theorem.

Theorem 2. For every $n \in \mathbb{Z}_{+}$the coupled dynamical system (7), (11) and (12) is Hamiltonian with respect to the Poisson structure $\mathcal{L}$ in the form (20) and the functional $\gamma_{n} \in I\left(\mathcal{G}^{*}\right)$.

Using the expression (18) one can construct the hierarchy of Hamiltonian evolution equations, describing commutative flows, generated by involutive with respect to the Lie-Poisson bracket (3) Casimir invariants $\gamma_{n} \in I\left(\mathcal{G}^{*}\right), n \in \mathbb{Z}_{+}$, on the extended space $\mathcal{G}^{*} \oplus W^{2 N \mid 2 N}$ at a fixed element $\tilde{l} \in \mathcal{G}^{*}$. For every $n \in \mathbb{Z}_{+}$the equation of such a type is equivalent to the system (7), (11) and (12).

\section{Hierarchies of additional symmetries}

The evolution type hierarchy (7), (11) and (12) possesses another set of invariants, which includes all natural powers of the eigenvalues $\lambda_{i}, i=\overline{1, N}$. They can be considered as smooth by Frechet functionals on the extended space $\mathcal{G}^{*} \oplus W^{2 N \mid 2 N}$ due to the representation:

$$
\lambda_{k}^{s}=\left\langle l^{s} f_{k}, f_{k}^{*}\right\rangle+\left\langle l^{s} \Phi_{k}, \Phi_{k}^{*}\right\rangle,
$$

where $s \in \mathbb{N}$, taking place for all $k=\overline{1, N}$ under the normalizing condition:

$$
\left\langle f_{k}, f_{k}^{*}\right\rangle+\left\langle\Phi_{k}, \Phi_{k}^{*}\right\rangle=1
$$

In the case of

$$
l:=l_{+}+\sum_{i=1}^{N}\left(f_{i} D_{\theta}^{-1} f_{i}^{*}+\Phi_{i} D_{\theta}^{-1} \Phi_{i}^{*}\right)
$$

the formula (21) leads to the following variation of the functionals $\lambda_{k}^{s} \in \mathcal{D}\left(\mathcal{G}^{*} \oplus W^{2 N \mid 2 N}\right)$, $k=\overline{1, N}$ :

$$
\begin{aligned}
\delta \lambda_{k}^{s}= & \left\langle\left(\delta l^{s}\right) f_{k}, f_{k}^{*}\right\rangle+\left\langle\left(\delta l^{s}\right) \Phi_{k}, \Phi_{k}^{*}\right\rangle \\
& +\left\langle l^{s}\left(\delta f_{k}\right), f_{k}^{*}\right\rangle+\left\langle l^{s} f_{k}, \delta f_{k}^{*}\right\rangle+\left\langle l^{s}\left(\delta \Phi_{k}\right), \Phi_{k}^{*}\right\rangle+\left\langle l^{s} \Phi_{k}, \delta \Phi_{k}^{*}\right\rangle
\end{aligned}
$$




$$
\begin{aligned}
= & \left(\delta l_{+}, M_{k}^{s}\right)+\sum_{i=1}^{N}\left(\left\langle\delta f_{i},\left(-M_{k}^{s}+\delta_{k}^{i} l^{s}\right)^{*} f_{i}^{*}\right\rangle+\left\langle\delta f_{i}^{*},\left(-M_{k}^{n}+\delta_{k}^{i} l^{s}\right) f_{i}\right\rangle\right. \\
& \left.+\left\langle\delta \Phi_{i},\left(-M_{k}^{s}+\delta_{k}^{i} l^{s}\right)^{*} \Phi_{i}^{*}\right\rangle+\left\langle\delta \Phi_{i}^{*},\left(-M_{k}^{n}+\delta_{k}^{i} l^{s}\right) \Phi_{i}\right\rangle\right),
\end{aligned}
$$

where $\delta_{k}^{i}$ is a Kronecker symbol and the operator $M_{k}^{s}, s \in \mathbb{N}$, is determined as

$$
M_{k}^{s}:=\sum_{p=0}^{s-1}\left(\left(l^{p} f_{k}\right) D_{\theta}^{-1}\left(l^{* s-1-p} f_{k}^{*}\right)+\left(l^{p} \Phi_{k}\right) D_{\theta}^{-1}\left(l^{* s-1-p} \Phi_{k}^{*}\right)\right)=\lambda_{k}^{s-1} M_{k}^{1} .
$$

Thus, one obtains the exact forms of gradients for the functionals $\lambda_{k}^{s} \in \mathcal{D}\left(\hat{\mathcal{G}}^{*} \oplus W^{2 N \mid 2 N}\right)$, $k=\overline{1, N}$ :

$$
\nabla \lambda_{k}^{s}\left(l_{+}, f_{i}, \Phi_{i}^{*}, f_{i}^{*}, \Phi_{i}\right)=\left(\begin{array}{c}
M_{k}^{s} \\
\left(-M_{k}^{s}+\delta_{k}^{i} l^{s}\right)^{*} f_{i}^{*} \\
\left(-M_{k}^{n}+\delta_{k}^{i} l^{s}\right) \Phi_{i} \\
\left(-M_{k}^{n}+\delta_{k}^{i} l^{s}\right) f_{i} \\
\left(-M_{k}^{s}+\delta_{k}^{i} l^{s}\right)^{*} \Phi_{i}^{*}
\end{array}\right),
$$

where $i=\overline{1, N}$. By means of the expression (23) the tensor product $\tilde{\mathcal{L}}$ of Poisson structures (13) and (14) generates the hierarchy of coupled evolution equations on $\mathcal{G}^{*} \oplus W^{2 N \mid 2 N}$ :

$$
\begin{aligned}
& d l_{+} / d \tau_{s, k}=-\left[M_{k}^{s}, \hat{l}_{+}\right]_{+}, \\
& d f_{i} / d \tau_{s, k}=\left(-M_{k}^{s}+\delta_{k}^{i} l^{s}\right) f_{i}, \quad d f_{i}^{*} / d \tau_{s, k}=\left(M_{k}^{s}-\delta_{k}^{i} l^{s}\right)^{*} f_{i}^{*}, \\
& d \Phi_{i} / d \tau_{s, k}=\left(-M_{k}^{s}+\delta_{k}^{i} l^{s}\right) \Phi_{i}, \quad d \Phi_{i}^{*} / d \tau_{s, k}=\left(M_{k}^{s}-\delta_{k}^{i} l^{s}\right)^{*} \Phi_{i}^{*},
\end{aligned}
$$

where $i=\overline{1, N}$, for every $k=\overline{1, N}$. Because of the Bäcklund transformation (19) the equation (24) is equivalent to the commutator relationship:

$$
d l / d \tau_{s, k}=-\left[M_{k}^{s}, l\right]=-\lambda_{k}^{s-1}\left[M_{k}^{1}, l\right]=\lambda_{k}^{s-1} d l / d \tau_{1, k},
$$

and the following theorem takes place:

Theorem 3. For every $k=\overline{1, N}$ and $s \in \mathbb{N}$ the coupled dynamical system (24), (25) and (26) is Hamiltonian one with respect to the Poisson structure $\mathcal{L}$ in the form (20) and the functional $\lambda_{k}^{n} \in \mathcal{D}\left(\mathcal{G}^{*} \oplus W^{2 N \mid 2 N}\right)$.

The coupled dynamical systems (24), (25) and (26) represent flows on $\mathcal{G}^{*} \oplus W^{2 N \mid 2 N}$, commuting one with each other.

Theorem 4. For $k=\overline{1, N}$ the coupled evolution equations (24), (25) and (26) form a set of additional symmetry hierarchies for the coupled dynamical system (7), (11) and (12).

Proof. To prove the theorem it is sufficient to show that

$$
\left[d / d t_{n}, d / d \tau_{1, k}\right]=0, \quad\left[d / d \tau_{1, k}, d / d \tau_{1, q}\right]=0,
$$

where $k, q=\overline{1, N}$ and $n \in \mathbb{N}$. The first equality in the formula (28) follows from the identities:

$$
d\left(\nabla \gamma_{n}(l)\right)_{+} / d \tau_{1, k}=\left[\left(\nabla \gamma_{n}(l)\right)_{+}, M_{1}^{1}\right]_{+}, \quad d M_{1}^{1} / d t_{n}=\left[\left(\nabla \gamma_{n}(\hat{l})\right)_{+}, M_{1}^{1}\right]_{-},
$$

the second one being a consequence of the relationship:

$$
d M_{k}^{1} / d \tau_{1, q}-d M_{q}^{1} / d \tau_{1, k}=\left[M_{k}^{1}, M_{q}^{1}\right] .
$$


When $N \geq 2$, a new class of nontrivial Hamiltonian flows $d / d T_{n, K}:=d / d t_{n}+\sum_{k=1}^{K} d / d \tau_{n, k}$, $n \in \mathbb{N}, K=\overline{1, N-1}$, in a Lax form on $\mathcal{G}^{*} \oplus W^{2 N \mid 2 N}$ can be constructed by use of the set of additional symmetry hierarchies for the Lie algebra of super-integro-differential operators. Acting on the functions $f_{i}, f_{i}^{*}, \Phi_{i}, \Phi_{i}^{*}, i=\overline{1, N}$, these flows generate $((1+K) \mid 1+1)$-dimensional supersymmetric nonlinear dynamical systems.

For the first time the additional symmetries in the case of $N=2$ were applied by E. Nissimov and S. Pacheva [13] to obtain a Lax integrable supersymmetric analog of the $(2+1)$-dimensional Davey-Stewartson system. If

$$
l:=\partial+f_{1} D_{\theta}^{-1} f_{1}^{*}+f_{2} D_{\theta}^{-1} f_{2}^{*}+\Phi_{1} D_{\theta}^{-1} \Phi_{1}^{*}+\Phi_{2} D_{\theta}^{-1} \Phi_{2}^{*} \in \mathcal{G}^{*},
$$

where $\left(f_{1}, f_{2}, \Phi_{1}^{*}, \Phi_{2}^{*}, f_{1}^{*}, f_{2}^{*}, \Phi_{1}, \Phi_{2}\right)^{T} \in W^{4 \mid 4}$, the flows $\partial / \partial \tau:=d / d \tau_{1,1}$ and $d / d T:=d / d T_{2,1}=$ $d / d t_{2}+d / d \tau_{2,1}$ on $\mathcal{G}^{*} \oplus W^{4 \mid 4}$, acting on the functions $f_{i}, f_{i}^{*}, \Phi_{i}, \Phi_{i}^{*}, i=1,2$, by the following way:

$$
\begin{array}{ll}
f_{1, \tau}=f_{1, x}+u_{1} f_{2}-\alpha_{1} \Phi_{2}, & f_{2, \tau}=-\bar{u}_{1} f_{1}+\bar{\alpha}_{2} \Phi_{1}, \\
f_{1, \tau}^{*}=f_{1, x}^{*}+\bar{u}_{1} f_{2}^{*}-\bar{\alpha}_{1} \Phi_{2}^{*}, & f_{2, \tau}^{*}=-u_{1} f_{1}^{*}-\alpha_{2} \Phi_{1}^{*}, \\
\Phi_{1, \tau}=\Phi_{1, x}-\alpha_{2} f_{2}+u_{2} \Phi_{2}, & \Phi_{2, \tau}=-\bar{\alpha}_{1} f_{1}-\bar{u}_{2} \Phi_{1}, \\
\Phi_{1, \tau}^{*}=\Phi_{1, x}^{*}-\bar{\alpha}_{2} f_{2}^{*}-\bar{u}_{2} \Phi_{2}^{*}, & \Phi_{2, \tau}^{*}=\alpha_{1} f_{1}^{*}-u_{2} \Phi_{1}^{*},
\end{array}
$$

and

$$
\begin{aligned}
& f_{1, T}=f_{1, x x}+f_{1, \tau \tau}+w_{1} D_{\theta} f_{1}+w_{0} f_{1}+2 v_{1, \tau} f_{1}-2 \beta_{\tau} \Phi_{1}, \\
& f_{2, T}=f_{2, x x}+w_{1} D_{\theta} f_{2}+w_{0} f_{2}-\bar{u}_{1} f_{1, \tau}+\bar{\alpha}_{2} \Phi_{1, \tau}+\bar{u}_{1, \tau} f_{1}-\bar{\alpha}_{2, \tau} \Phi_{1, \tau}, \\
& f_{1, T}^{*}=-f_{1, x x}^{*}-f_{1, \tau \tau}^{*}-D_{\theta}\left(w_{1} f_{1}^{*}\right)-w_{0} f_{1}^{*}-2 v_{1, \tau} f_{1}^{*}+2 \bar{\beta}_{\tau} \Phi_{1}^{*}, \\
& f_{2, T}^{*}=-f_{2, x x}^{*}-D_{\theta}\left(w_{1} f_{2}^{*}\right)-w_{0} f_{2}^{*}+u_{1} f_{1, \tau}^{*}+\alpha_{2} \Phi_{1, \tau}^{*}-u_{1, \tau} f_{1}^{*}+\alpha_{2, \tau} \Phi_{1, \tau}^{*}, \\
& \Phi_{1, T}=\Phi_{1, x x}+\Phi_{1, \tau \tau}+w_{1} D_{\theta} \Phi_{1}+w_{0} \Phi_{1}+2 f_{1} \bar{\beta}_{\tau}+2 v_{2, \tau} \Phi_{1}, \\
& \Phi_{2, T}=\Phi_{2, x x}+w_{1} D_{\theta} \Phi_{2}+w_{0} \Phi_{2}-\bar{\alpha}_{1} f_{1, \tau}-\bar{u}_{2} \Phi_{1, \tau}+\bar{\alpha}_{1, \tau} f_{1}+\bar{u}_{2, \tau} \Phi_{1, \tau}, \\
& \Phi_{1, T}^{*}=-\Phi_{1, x x}^{*}-\Phi_{1, \tau \tau}-D_{\theta}\left(w_{1} \Phi_{1}^{*}\right)-w_{0} \Phi_{1}^{*}-2 f_{1}^{*} \beta_{\tau}-2 v_{2, \tau} \Phi_{1}^{*}, \\
& \Phi_{2, T}^{*}=-\Phi_{2, x x}^{*}-D_{\theta}\left(w_{1} \Phi_{2}^{*}\right)-w_{0} \Phi_{2}^{*}-\alpha_{1} f_{1, \tau}^{*}+u_{2} \Phi_{1, \tau}^{*}+\alpha_{1, \tau} f_{1}^{*}-u_{2, \tau} \Phi_{1, \tau}^{*}, \\
& D_{\theta} u_{1}=f_{1} f_{2}^{*}, \quad D_{\theta} u_{2}=\Phi_{1} \Phi_{2}^{*}, \quad D_{\theta} \bar{u}_{1}=f_{1}^{*} f_{2}, \quad D_{\theta} \bar{u}_{2}=\Phi_{1}^{*} \Phi_{2}, \\
& D_{\theta} v_{1}=f_{1} f_{1}^{*}, \quad D_{\theta} v_{2}=\Phi_{1} \Phi_{1}^{*}, \quad D_{\theta} \alpha_{1}=f_{1} \Phi_{2}^{*}, \quad D_{\theta} \alpha_{2}=\Phi_{1} f_{2}^{*}, \\
& D_{\theta} \bar{\alpha}_{1}=f_{1}^{*} \Phi_{2}, \quad D_{\theta} \bar{\alpha}_{2}=\Phi_{1}^{*} f_{2}, \quad D_{\theta} \beta=f_{1} \Phi_{1}^{*}, \quad D_{\theta} \bar{\beta}=f_{1}^{*} \Phi_{1},
\end{aligned}
$$

where $\left(\nabla \gamma_{2}(l)\right)_{+}:=\partial^{2}+w_{1} D_{\theta}+w_{0}$, represent $(2 \mid 1+1)$-dimensional supersymmetric nonlinear dynamical system. The system (29) and (4) possesses an infinite sequence of local conservation laws, which can be found by the formula (6), and a Lax representation, given by the spectral problem (8) and the evolution equations:

$$
\begin{aligned}
& f_{\tau}=-M_{1}^{1} f, \\
& f_{T}=\left(\left(\nabla \gamma_{2}(l)\right)_{+}-M_{1}^{2}\right) f,
\end{aligned}
$$

for an arbitrary eigenfunction $f \in W^{1 \mid 0}$ or $f \in W^{0 \mid 1}$. The relationships and (32) lead to additional nonlinear constraints such as

$$
\begin{aligned}
& w_{0, \tau}=2 w_{1}\left(f_{1} f_{1}^{*}-\Phi_{1} \Phi_{1}^{*}\right)+2\left(f_{1}\left(D_{\theta} f_{1}^{*}\right)+\Phi_{1}\left(D_{\theta} \Phi_{1}^{*}\right)\right)_{x}, \\
& w_{1, \tau}=-2\left(f_{1} f_{1}^{*}\right)_{x}+2\left(\Phi_{1} \Phi_{1}^{*}\right)_{x} .
\end{aligned}
$$


When $f_{1}:=\psi, f_{1}^{*}:=\theta \psi^{*}, f_{2}=f_{2}^{*}=0$ and $\Phi_{1}=\Phi_{1}^{*}=\Phi_{2}=\Phi_{2}^{*}=0$, the equations and are reduced to the Lax integrable $(2+1)$-dimensional Davey-Stewartson system $[5,16,17]$ :

$$
\begin{aligned}
& \psi_{1, T}=\psi_{1, x x}+\psi_{1, \tau \tau}+2\left(S-2 \psi \psi^{*}\right) \psi, \\
& \psi_{1, T}^{*}=-\psi_{1, x x}^{*}-\psi_{1, \tau \tau}^{*}-2\left(S-2 \psi \psi^{*}\right) \psi^{*}, \\
& S_{x \tau}=(\partial / \partial x+\partial / \partial \tau)^{2} \psi \psi^{*},
\end{aligned}
$$

where $2 S:=w_{0}^{0}+2 v_{1, \tau}^{0}+4 \psi \psi^{*}, w_{0}:=w_{0}^{0}, v_{1, \tau}:=v_{1, \tau}^{0}$ and $\psi, \psi^{*} \in L_{\infty}\left(\mathbb{S}^{1} ; \mathbb{C}\right)$.

The Lax representation (10), (4) and (32) for the $(2 \mid 1+1)$-dimensional supersymmetric nonlinear dynamical Davey-Stewartson system (29), (4) and (4) has equivalent matrix form:

$$
\begin{aligned}
& D_{\theta} F=\left(\begin{array}{cccccc}
0 & 0 & 0 & 0 & 0 & 1 \\
f_{1}^{*} & 0 & 0 & 0 & 0 & 0 \\
f_{2}^{*} & 0 & 0 & 0 & 0 & 0 \\
\Phi_{1}^{*} & 0 & 0 & 0 & 0 & 0 \\
\Phi_{2}^{*} & 0 & 0 & 0 & 0 & 0 \\
\lambda & -f_{1} & -f_{2} & -\Phi_{1} & -\Phi_{2} & 0
\end{array}\right) F \\
& \frac{d F}{d \tau}=\left(\begin{array}{cccccc}
0 & -f_{1} & 0 & -\Phi_{1} & 0 & 0 \\
D_{\theta} f_{1}^{*} & -\lambda & \bar{u}_{1} & 0 & \bar{\alpha}_{1} & -f_{1}^{*} \\
0 & -u_{1} & 0 & \alpha_{2} & 0 & 0 \\
D_{\theta} \Phi_{1}^{*} & 0 & \bar{\alpha}_{2} & -\lambda & \bar{u}_{2} & \Phi_{1}^{*} \\
0 & -\alpha_{1} & 0 & -u_{2} & 0 & 0 \\
\Phi_{1} \Phi_{1}^{*}-f_{1} f_{1}^{*} & -D_{\theta} f_{1} & 0 & -D_{\theta} \Phi_{1} & 0 & 0
\end{array}\right) F, \\
& \frac{d F}{d T}=C F \text {, }
\end{aligned}
$$

where $F=\left(F^{0}:=f, F^{2}, F^{4}, F^{1}, F^{3}, F^{5}\right)^{\tau} \in W^{3 \mid 3}, C:=\left(C_{m n}\right) \in \operatorname{gl}(3 \mid 3), m, n=\overline{1,6}$, and

$$
\begin{aligned}
& C_{11}=\lambda^{2}+\frac{1}{2} w_{0}+f_{1} D_{\theta} f_{1}^{*}+\Phi_{1} D_{\theta} \Phi_{1}^{*}, \quad C_{12}=-\left(2 \lambda f_{1}+f_{1, x}+f_{1, \tau}\right), \\
& C_{13}=-\left(\lambda f_{2}+f_{2, x}\right)+\bar{u}_{1} f_{1}-\bar{\alpha}_{2} \Phi_{1}, \quad C_{14}=-\left(2 \lambda \Phi_{1}+\Phi_{1, x}+\Phi_{1, \tau}\right), \\
& C_{15}=-\left(\lambda \Phi_{2}+\Phi_{2, x}\right)-\bar{u}_{2} \Phi_{1}+\bar{\alpha}_{1} f_{1}, \quad C_{16}=\frac{1}{2} w_{1}-f_{1} f_{1}^{*}+\Phi_{1} \Phi_{1}^{*}, \\
& C_{21}=-w_{1} f_{1}^{*}+2 D_{\theta}\left(-f_{1, x}^{*}+\lambda f_{1}^{*}\right)-\bar{u}_{1} D_{\theta} f_{2}^{*}-\bar{\alpha}_{1} D_{\theta} \Phi_{2}^{*}, \\
& C_{22}=-\lambda^{2}-2 D_{\theta}\left(f_{1} f_{1}^{*}\right)-u_{1} \bar{u}_{1}+\alpha_{1} \bar{\alpha}_{1}, \quad C_{23}=-D_{\theta}\left(f_{2} f_{1}^{*}\right)+\lambda \bar{u}_{1}-\bar{u}_{1, \tau}, \\
& C_{24}=-2 D_{\theta}\left(\Phi_{1} f_{1}^{*}\right)+\bar{u}_{1} \alpha_{2}-u_{2} \bar{\alpha}_{1}, \quad C_{25}=-D_{\theta}\left(\Phi_{2} f_{1}^{*}\right)+\lambda \bar{\alpha}_{1}-\bar{\alpha}_{1, \tau}, \\
& C_{26}=2\left(-\lambda f_{1}^{*}+f_{1, x}^{*}\right)+\bar{u}_{1} f_{2}^{*}-\bar{\alpha}_{1} \Phi_{2}^{*}, \\
& C_{31}=-\frac{1}{2} w_{1} f_{2}^{*}+D_{\theta}\left(-f_{2, x}^{*}+\lambda f_{2}^{*}\right)+u_{1} D_{\theta} f_{1}^{*}+\alpha_{2} D_{\theta} \Phi_{1}^{*}, \\
& C_{32}=-D_{\theta}\left(f_{1} f_{2}^{*}\right)-\lambda u_{1}-u_{1, \tau}, \quad C_{33}=-D_{\theta}\left(f_{2} f_{2}^{*}\right)+u_{1} \bar{u}_{1}-\alpha_{2} \bar{\alpha}_{2}, \\
& C_{34}=-D_{\theta}\left(\Phi_{1} f_{2}^{*}\right)+\lambda \alpha_{2}+\alpha_{2, \tau}, \quad D_{\theta}\left(\Phi_{2} f_{2}^{*}\right)+u_{1} \bar{\alpha}_{1}-\bar{u}_{2} \alpha_{2}, \\
& C_{36}=\left(-\lambda f_{2}^{*}+f_{2, x}^{*}\right)-u_{1} f_{1}^{*}+\alpha_{2} \Phi_{1}^{*}, \\
& C_{41}=-w_{1} \Phi_{1}^{*}+2 D_{\theta}\left(-\Phi_{1, x}^{*}+\lambda \Phi_{1}^{*}\right)+\bar{u}_{2} D_{\theta} \Phi_{2}^{*}-\bar{\alpha}_{2} D_{\theta} f_{2}^{*}, \\
& C_{42}=-2 D_{\theta}\left(f_{1} \Phi_{1}^{*}\right)-u_{1} \bar{\alpha}_{2}-\bar{u}_{2} \alpha_{1}, \quad C_{43}=-D_{\theta}\left(f_{2} \Phi_{1}^{*}\right)+\lambda \bar{\alpha}_{2}-\bar{\alpha}_{2, \tau}, \\
& C_{44}=-\lambda^{2}-2 D_{\theta}\left(\Phi_{1} \Phi_{1}^{*}\right)-u_{2} \bar{u}_{2}-\alpha_{2} \bar{\alpha}_{2}, \quad C_{45}=-D_{\theta}\left(\Phi_{2} \Phi_{1}^{*}\right)+\lambda \bar{u}_{2}-\bar{u}_{2, \tau}, \\
& C_{46}=2\left(-\lambda \Phi_{1}^{*}+\Phi_{1, x}^{*}\right)+\bar{u}_{2} \Phi_{2}^{*}+\bar{\alpha}_{2} f_{2}^{*}, \\
& C_{51}=-\frac{1}{2} w_{1} \Phi_{2}^{*}+D_{\theta}\left(-\Phi_{2, x}^{*}+\lambda \Phi_{2}^{*}\right)+\alpha_{1} D_{\theta} f_{1}^{*}+u_{2} D_{\theta} \Phi_{1}^{*},
\end{aligned}
$$




$$
\begin{aligned}
C_{52}= & -D_{\theta}\left(f_{1} \Phi_{2}^{*}\right)-\lambda \alpha_{1}-\alpha_{1, \tau}, \quad C_{53}=-D_{\theta}\left(f_{2} \Phi_{2}^{*}\right)+\alpha_{1} \bar{u}_{1}-\bar{\alpha}_{2} u_{2}, \\
C_{54}= & -D_{\theta}\left(\Phi_{1} \Phi_{2}^{*}\right)-\lambda u_{2}-u_{2, \tau}, \quad C_{55}=-D_{\theta}\left(\Phi_{2} \Phi_{2}^{*}\right)+\alpha_{1} \bar{\alpha}_{1}+u_{2} \bar{u}_{2}, \\
C_{56}= & \left(-\lambda \Phi_{2}^{*}+\Phi_{2, x}^{*}\right)-\alpha_{1} f_{1}^{*}+u_{2} \Phi_{1}^{*}, \\
C_{61}= & \frac{1}{2} D_{\theta} w_{0}+\left(D_{\theta} f_{1}\right) D_{\theta} f_{1}^{*}+\left(D_{\theta} \Phi_{1}\right) D_{\theta} \Phi_{1}^{*} \\
& -\left(f_{1, \tau} f_{1}^{*}+f_{2, x} f_{2}^{*}-\Phi_{1, \tau} \Phi_{1}^{*}-\Phi_{2, x} \Phi_{2}^{*}\right)+\bar{u}_{1} f_{1} f_{2}^{*}-\bar{\alpha}_{1} f_{1} \Phi_{2}^{*}+\bar{\alpha}_{2} \Phi_{1} f_{2}^{*}+\bar{u}_{2} \Phi_{1} \Phi_{2}^{*}, \\
C_{62}= & -D_{\theta}\left(2 \lambda f_{1}+f_{1, x}+f_{1, \tau}\right)+\frac{1}{2} w_{1} f_{1}+f_{1}\left(-f_{1} f_{1}^{*}+\Phi_{1} \Phi_{1}^{*}\right), \\
C_{63}= & -D_{\theta}\left(\lambda f_{2}+f_{2, x}\right)+\frac{1}{2} w_{1} f_{2}+\bar{u}_{1}\left(D_{\theta} f_{1}\right)+\bar{\alpha}_{2}\left(D_{\theta} \Phi_{1}\right) \\
C_{64}= & -D_{\theta}\left(2 \lambda \Phi_{1}+\Phi_{1, x}+\Phi_{1, \tau}\right)+\frac{1}{2} w_{1} \Phi_{1}-f_{1} f_{1}^{*} \Phi_{1}, \\
C_{65}= & -D_{\theta}\left(\lambda \Phi_{2}+\Phi_{2, x}\right)+\frac{1}{2} w_{1} \Phi_{2}-\bar{u}_{2} D_{\theta} \Phi_{1}-\bar{\alpha}_{1} D_{\theta} f_{1}, \\
C_{66}= & \lambda^{2}+\frac{1}{2} w_{0}+\frac{1}{2} D_{\theta} w_{1}-\left(D_{\theta} f_{1}\right) f_{1}^{*}+\left(D_{\theta} \Phi_{1}\right) \Phi_{1}^{*} .
\end{aligned}
$$

In fact, one has found a triple matrix linearization for a $(2 \mid 1+1)$-dimensional dynamical system, that is important for the standard method of inverse scattering transformation [3] as well as for the reduction procedure $[18,19]$ upon invariant subspaces of associated spectral problem eigenvalues.

The method of additional symmetries is effective for constructing a wide class of $(2 \mid 1+1)$ dimensional supersymmetric nonlinear dynamical systems with a triple matrix linearization.

\section{Conclusion}

By now several regular Lie-algebraic approaches existed to constructing Lax integrable $(2+1)$ dimensional nonlinear dynamical systems on functional manifolds, which were presented in [12, 20, 21, 22]. In this paper a new Lie-algebraic method is devised for introducing one more commuting variable into $(1 \mid 1+1)$-dimensional dynamical systems with preserving their integrability by Lax. It involves use of additional symmetries [13] for a Hamiltonian flow hierarchy on extended dual space to some operator Lie algebra.

Any integrable $(2 \mid 1+1)$-dimensional supersymmetric nonlinear dynamical system obtained by means of the method possesses an infinite sequence of local conservation laws and a triple matrix linearization of a Lax type. These properties make it possible to apply the standard inverse scattering transformation [3] and the reduction procedure $[18,19]$ upon invariant subspaces.

If $N>2$ in the representation (22), the hierarchies of additional symmetries can be used for constructing Lax integrable $((1+K) \mid 1+1)$-dimensional supersymmetric systems, where $K=\overline{1, N-1}$.

Analyzing the structure of the Bäcklund type transformation (19) as a key point of the method, one can observe that it strongly depends on an ad-invariant scalar product chosen for an operator Lie algebra $\mathcal{G}$ and a Lie algebra decomposition like (2). Since there are other possibilities of choosing ad-invariant scalar products on $\mathcal{G}$ and such decompositions, they give rise naturally to other Bäcklund transformations.

\section{Acknowledgements}

The author thanks Professor A.K. Prykarpatsky for useful discussions and Organizers of Sixth International Conference "Symmetry in Nonlinear Mathematical Physics" (2005, Kyiv) for invitation to take part in the conference. The present paper is the written version of the talk delivered by the author at this conference. 
[1] Adler M., On a trace functional for formal pseudo-differential operators and the symplectic structures of a Korteweg-de Vries equation, Invent. Math., 1979, V.50, N 2, 219-248.

[2] Lax P.D., Periodic solutions of the KdV equation, Comm. Pure Appl. Math., 1975, V.28, 141-188.

[3] Novikov S.P. (Editor), Soliton theory: method of the inverse problem, Moscow, Nauka, 1980 (in Russian).

[4] Prykarpatsky A.K., Mykytiuk I.V., Algebraic integrability of nonlinear dynamical systems on manifolds: classical and quantum aspects, Dordrecht-Boston-London, Kluwer Academic Publishers, 1998.

[5] Blaszak M., Multi-Hamiltonian theory of dynamical systems, Verlag-Berlin-Heidelberg, Springer, 1998.

[6] Manin Yu.I., Radul A.O., A supersymmetric extension of the Kadomtsev-Petviashvili hierarchy, Comm. Math. Phys., 1985, V.98, 65-77.

[7] Oevel W., Popowicz Z., The bi-Hamiltonian structure of fully supersymmetri Korteweg-de Vries systems, Comm. Math. Phys., 1991, V.139, 441-460.

[8] Semenov-Tian-Shansky M.A., What is the $\mathcal{R}$-matrix, Funct. Anal. Appl., 1983, V.17, N 4, 17-33 (in Russian).

[9] Oevel W., R-structures, Yang-Baxter equations and related involution theorems, J. Math. Phys., 1989, V.30, N 5, 1140-1149.

[10] Oevel W., Strampp W., Constrained KP hierarchy and bi-Hamiltonian structures, Comm. Math. Phys., 1993, V.157, 51-81.

[11] Prykarpatsky Ya.A., Structure of integrable Lax flows on nonlocal manifolds: dynamical systems with sources, Math. Methods and Phys.-Mech. Fields, 1997, V.40, N 4, 106-115 (in Ukrainian).

[12] Prykarpatsky A.K., Hentosh O.Ye., The Lie-algebraic structure of $(2+1)$-dimensional Lax type integrable nonlinear dynamical systems, Ukrainian Math. J., 2004, V.56, N 7, 939-946.

[13] Nissimov E., Pacheva S., Symmetries of supersymmetric integrable hierarchies of KP type, J. Math. Phys., 2002, V.43, N 5, 2547-2586; nlin.SI/0102010.

[14] Berezin F.A., Introduction to algebra and analysis with anticommuting variables, Moscow Univ., 1983 (in Russian).

[15] Shander V.N., Analogues of the Frobenius and Darboux theorems, Reports of Bulgarian Academy of Sciences, 1983, V.36, N 3, 309-311.

[16] Ablowitz M.J., Segur H., Solitons and the inverse scattering transform, Philadelphia, SIAM, 1981.

[17] Kulish P.P., Lipovsky V.D., On Hamiltonian interpretation of the inverse problem method for the DaveyStewartson equation, LOMI Proceedings, Leningrad, Nauka, 1987, V.161, 54-71 (in Russian).

[18] Prykarpatsky A.K., Hentosh O.E., Blackmore D.L., The finite-dimensional Moser type reductions of modified Boussinesq and super-Korteweg-de Vries Hamiltonian systems via the gradient-holonomic algorithm and the dual moment maps. I, J. Nonlinear Math. Phys., 1997, V.4, N 3-4, 455-469.

[19] Prykarpatsky A.K., Blackmore D., Strampp W., Sydorenko Yu., Samuliak R., Some remarks on Lagrangian and Hamiltonian formalism, related to infinite-dimensional dynamical systems with symmetries, Condensed Matter Phys., 1995, N 6, 79-104.

[20] Sato M., Soliton equations as dynamical systems on infinite Grassmann manifolds, RIMS Kokyuroku, Kyoto Univ., 1981, V.439, 30-40.

[21] Reiman A.G., Semenov-Tian-Shansky M.A., The Hamiltonian structure of Kadomtsev-Petviashvili type equations, LOMI Proceedings, Leningrad, Nauka, 1987, V.164, 212-227 (in Russian).

[22] Prykarpatsky A.K., Samoilenko V.Hr., Andrushkiw R.I., Mitropolsky Yu.O., Prytula M.M., Algebraic structure of the gradient-holonomic algorithm for Lax integrable nonlinear systems. I, J. Math. Phys., 1994, V.35, N 4, 1763-1777. 